\title{
WHAT WE DON'T KNOW ABOUT STARSPOTS
}

\author{
DOUGLAS S. HALL \\ Dyer Observatory \\ Vanderbilt University \\ Nashville, Tennessee 37235, U.S.A.
}

\section{Introduction}

The scope of this paper will be starspots, and by that I mean regions cooler than the surrounding photosphere (like sunspots) but I also mean the whole range from very small (like sunspots) to very large (such as seen on very active stars like RS CVn).

The focus will be on what we don't understand, or understand incompletely. I'll consider "understanding" at the empirical or phenomenonological level and at the theoretical level in terms of the underlying physics.

There will be a list of 13 questions. This will be a quite personal perspective, based on progress we have made since I saw my first giant starspot in 1972 (Hall 1972). At that time the one big question was the reality of giant starspots, and there was no understanding of them at all. Perhaps some of these 13 questions are understood better than I realize, and I must have missed something important in the literature, which since 1972 has become incredibly extensive. Moreover, some may have been answered just as a result of this symposium, but I have not tried to update my talk or this paper after reading all of the printed abstracts and viewing all of the posters. As an epilogue, though, let me draw your attention to a paper entitled "Random Spots on Chromospherically Active Stars" submitted recently to ApJ Letters by my colleagues J. A. Eaton, G. W. Henry, and F. C. Fekel. In my judgement this is an extremely valuable contribution with the potential to resolve some of the questions I am asking in this paper.

Earlier (at the 1979 I.A.U. General Assembly in Montreal, in a Joint Meeting of Commissions 10, 27, 40,42,44, and 48) I gave an invited talk entitled "A Baker's Dozen: 13 Unsolved Problems with the RS Canum 
Venaticorum and BY Draconis Stars" (Hall 1980). Now, 16 years later, the scope will be narrower, just the starspots.

The basis for much of my perspective is questions left unanswered in my mind after preparing the paper "Starspot Evolution, Differential Rotation, and Magnetic Cycles in the Chromospherically Active Binaries lambda Andromedae, sigma Geminorum, II Pegasi, and V711 Tauri" (Henry, Eaton, Hamer, and Hall 1995). It can be used for most of the pertinent references not cited explicitly in this paper.

\section{How much can we trust starspot maps?}

There are basically three approaches to starspot mapping: Doppler imaging, light curve inversion, and eclipsing binaries.

With respect to Doppler imaging, several strategies are employed by different investigators. When these different strategies have been applied to identical data sets, some of the resulting maps are generally consistent but different in detail. In some cases, however, the maps are very different.

A light curve, even with the same signal-to-noise ratio, will necessarily be a poorer source of information than Doppler imaging. This is because input from Doppler imaging consists of intensity versus radial velocity and time, whereas input from a light curve is only intensity versus time. Moreover, a light curve cannot reveal the presence of a polar spot, which produces no rotational modulation. Another deficiency is that most modelling of light curves to date has been restricted to determining the size, latitude, and longitude of two (or maybe three) round or square spots. This has been a sensible approximation to make, because determining more than about six parameters generally is not warranted by the data. Some of these two-spot fits have been determined rigorously, for example, by least squares. Others have been done by trial and error. Recently (Wild 1994, Drish et al. 1995) formal inversion techniques which include a maximum entropy feature have been developed. Such techniques do away with the limitations of two- spot arbitrariness and promise to squeeze out the maximum information contained within a given light curve. These light curve inversion techniques have not yet been applied to real data, i.e., light curves of specific spotted stars.

Eclipsing binaries, in which a spotted star is eclipsed by the other star, can be useful in providing an independent handle on the mapping problem. Alone, this technique must yield an incomplete map, because only one hemisphere is scanned, and only a strip of restricted width in latitude. This potentially valuable approach has been used very seldom to date, in only a few eclipsing binaries and by only a few investigators.

In light of the lingering uncertainties with a map determined by any one 
technique and the deficiencies inherent in any one technique, it would help enormously to compare and/or combine information derived from Doppler imaging and light curves and eclipsing binaries, using the same star and data taken contemporaneously. This has been done in a few cases, but precious few.

\section{How big are starspots?}

Maps from Doppler imaging show small and medium and large spots, as well as polar spots, sometimes on the same star at the same epoch. A question is, can we believe the reality of the smallest ones, i.e., can we establish a minimum spot size. Let me make clear the larger question here. It has been shown (Hall 1991b, 1994) that there are two types of convective stars: (1) inactive or weak-dynamo stars (like the Sun) with little spots only and (2) active or strong-dynamo stars with big spots. The larger question is, do the stars with big spots also have small spots or do they have only big spots.

Light curve analysis, using two-spot models, reveals medium and big spots but are cannot reveal small spots like sunspots. The big ones can be really big, a dark area covering half of a visible hemisphere. II Peg, with a light curve amplitude in 1986 of 0.5 magnitude in $\mathrm{V}$, was the record holder for a while (Doyle et al. 1988), until replaced by XX Tri, with an amplitude in 1990 of 0.6 magnitude in V (Nolthenius 1991, Hampton et al. 1995). The question here is, what is the nature of such a large dark region. (1) Is it one huge spot, with a giant umbra surrounded by a giant penumbra? (2) Is it a huge spot pair, with magnetically North and South components? (3) Is it several medium-sized spots grouped together, perhaps temporarily? (4) Is is many small spots (perhaps as small as sunspots) clumped together?

\section{Starspot temperatures}

There have been many determinations of the temperature in dark regions, by several techniques. Each one, however, typically has been a single value. A single value must be some sort of weighted average of all spots on a visible hemisphere at a given time and also some sort of weighted average of the two temperatures in the spots, if they have an umbra-penumbra structure. Moreover, not many stars have had a spot temperature determination more than once.

So we are let naturally to pose the following three questions: (1) Are there different temperatures for different spots, if several are present at the same time? (2) Are two temperatures needed for each spot, i.e., is there an umbra- penumbra structure? (3) What is the evolution in temperature, as a large spot emerges and vanishes? Even a null result here would be a contribution. 


\section{Strong magnetic fields}

There have been several believable determinations of strong (several $\mathrm{kG}$ ) magnetic fields in stars known to have heavily spotted surfaces. This finding was important historically, in finally convincing the last skeptics that large starspots really do exist.

Precious few magnetic field strength determinations, however, have been done in such a way as to enable a comparison to be made with contemporaneous maps of photospheric surface brightness distribution, to see how magnetic parameters are correlated spatially and temporally with known spotted regions.

\section{Latitude extent}

We need to know (1) which stars have only low-latitude spots, like the Sun does, (2) which stars have spots at all latitudes, up to the pole, and (3) which stars have only polar or high-latitude spots. By "which stars" I mean which types of stars, as characterized by rotation rate, depth of convection, spectral type, evolutionary state, etc.

Some statements have been made in answer to this question, on a starby-star basis, and a few tentative general statements. Some predictions have been made based of theoretical calculations, comparing the competition between coriolis forces and magnetic bouyancy, but most of these theoretical predictions are incomplete and some are contradictory. We need (and don't yet have) a complete picture, theory with the predictive power to determine latitude extent as it is observed on real stars.

\section{Surface differential rotation}

We know that the Sun's rotation rate decreases from equator to pole, following a $\sin ^{2} \theta$ dependence. What do other stars do? Theoreticians tell us there are many possibilities: (1) The magnitude of the gradient, usually characterized by the coefficient $k$, can be more or less than the Sun's $k=$ 0.18 . (2) The algebraic sense can be opposite, faster at higher latitudes. (3) The functional dependence on latitude can be double-valued, with a maximum or minimum rotation rate at some intermediate latitude.

With respect to the algebraic sense, Doppler imaging has found some stars with a gradient opposite from the Sun. Such results, however, are far too fragmentary for us to know which types of stars do what.

With respect to the magnitude, light curve analysis of a much larger sample of spotted stars has evaluated the magnitude of the gradient. The most extensive such sample (Hall 1991b) shows a linear relation between $\log k$ and $\log P($ rot $)$, in the sense that $k$ decreases and approaches rigid- 
body rotation for very short rotation periods. This behavior supposedly is the opposite of what is expected from linear dynamo theory. If so, then we must ask, why is it behaving this way?

I myself first displayed this relation five years ago (Hall 1991b) and an earlier version of the same relation, based on fewer stars, was shown by Rodonó (1986) even before that, but an explanation of what this means has yet to materialize, or else I have been overlooking an important reference.

\section{Permanent active-longitude structure on spotted stars ?}

Active-longitude structure, as it occurs on the Sun and is suspected of occurring on other stars, takes the form of four quadrants: active, inactive, active, inactive.

It is generally acknowledged that there are long-lived active-longitude structures on the Sun, as defined by the global magnetic field and by the occurrence of energetic flares.

Several investigators report finding similar structure on several spotted stars, but the case is not convincing to a moderate skeptic, for a variety of reasons.

First, an active longitude is supposed to be defined by the longitude of first emergence of all spots, yet surface differential rotation will naturally carry a spot away from its point of origin, perhaps into the adjacent inactive quadrant. Consequently, a responsible investigation must fix the longitude of first emergence for each spot, and that is not easy to do with confidence or precision.

Second, in many of the cases reported so far, the statistics are a bit skaky.

Third, quadrant sector has not been found on all spotted stars which have been investigated for quadrant structure.

Fourth, although we should not distrust an observation just because it has not been predicted by theory, it still would be reassuring to know if we should be expecting quadrant structure a priori. I have encountered a few cases where some form of dynamo theory might imply some sort of long-lived longitude structure, but these cases have been few, tentative, and inconsistent.

\section{Orientation of quadrant structure in a binary}

If there is quadrant structure and if a spotted star has a binary companion, the question then is, how does the quadrant structure orient itself with respect to the line of centers?

There have been some findings which address this question. In some binaries the active quadrants coincide with the two hemispheres visible 
during the binary's quadratures (Zeilik 1992). In other binaries the active quadrants coincide with the hemispheres visible during the binary's conjunctions. In several binaries the orientation is intermediate between the quadratures and the conjunctions. In a few cases there appears to be only one active quadrant, not two. And, to repeat a point made in the previous section, the spotted stars in some binaries do not appear to have any quadrant structure at all.

At best, the picture painted by all of these findings is a confusing picture. At worst, they make a skeptic ask if maybe there is no preferred orientation with respect to the line of centers in a binary.

\section{Correlation with other signatures of activity ?}

By "signatures" of chromospheric activity, I mean spots on the photosphere, emission in the $\mathrm{Ca}$ II $\mathrm{H}$ and $\mathrm{K}$ lines from plages in the chromosphere, $\mathrm{H}$ alpha in emission, emission in extreme ultraviolet lines from the transition region, x-ray emission from the hot corona, and radio emission from the outer corona and/or the wind. The question is, is there spatial and temporal correlation between photospheric spots and the other signatures of chromospheric activity? The answer, at hand already, is "yes and no".

Several problems make this a difficult question to answer. (1) There is a shortage of synoptic data simultaneously in all signatures, except for a few stars at a few epochs, for which the campaign organizers should be commended. (2) Activity in several of the signatures is inherently sporadic, flare-like in nature. This makes establishing a correlation statistically difficult. (3) Some signatures may be temporally coincident but not spatially coincident, i.e., there may be an offset, in longitude, for example. (4) Some signatures seem not to be correlated at all, and establishing an absence of correlation is the more difficult task.

Despite all of this, a picture is emerging. In the third dimension (moving radially out from the photospheric surface) we go from good correlation to no correlation. At one extreme, closest in, we find $\mathrm{Ca}$ II $\mathrm{H}$ and $\mathrm{K}$ emission (plages) correlated best with starspots. At the other extreme, farthest out, we find radio emission (from the outer corona) not correlated with starspots at all.

I am pleased that we have learned so much here, but the picture does need to be cleaned up and a few instances of apparently contradictory evidence needs to be resolved. Let me close by drawing attention to a provocative finding, made almost ten years ago but apparently ignored. Glebocki et al. (1986) found that $\mathrm{Mg}$ II $\mathrm{h}$ and k emission intensity (from the transition region) was not correlated with the 54-day rotation period in the asynchronously rotating spotted star in the spectroscopic binary lambda 
And, but was correlated with the 20.5-day orbital period. This remarkable finding must be worked into the picture somehow, before the picture can be considered complete.

\section{What are spot lifetimes?}

An answer to this question has been determined empirically (Hall and Henry 1994) by analysis of several dozen spotted stars with decade-long continuous photometric coverage. They find that the lifetimes of relatively small spots are proportional to their sizes, consistent with and probably an extension of the similar relation which applies to sunspots, and that the lifetimes of relatively large spots are equal to the time required for them to be disrupted by the shear of surface differential rotation, without claiming that differential rotation necessarily is the mechanism determining their lifetimes.

It bothers me that this is the only published answer. It would be reassuring to see it confirmed by other investigators, independently analyzing the same photometric material or, better, analyzing new photometry of the same and/or different spotted stars.

\section{What determines large starspot lifetimes ?}

What is the physical mechanism for creating, maintaining, and dissolving starspots, especially the largest spots. We stress the largest spots because in them we have a phenomenon for which the solar analogy may fail to provide guidance. Pursuing a non-solar analog might lead us to new physics. To steer the search for an answer, let me ask the question in three parts.

Do all starspots, large or small, erupt from some depth. If so, from what depth. This question is basically equivalent to the debate between the distributive dynamo and the convective/radiative interface as the site of the underlying magnetic field.

Do little spots (once formed, by whatever mechanism causes little spots to form) clump together to make a big spot? If so, then what is the clumping force? Is it thermal (the ice-cubes-in-a-bath-tub phenomenon)? Is it magnetic? Is it random?

Once formed, is a big spot free to drift in response to surface differential rotation? Similarly, are all parts of a large spot free to be disrupted by surface differential rotation? If so, then specifically what has happened? Does it break loose from its magnetic anchor? If it can only stretch or bend its mooring lines, how much can they stretch or bend? Does the same pattern of surface differential rotation extend downward to the deeper layer where the magnetic anchors lie? 


\section{The physical determinant of spot sizes}

To first order, spot sizes seem to be determined by Rossby number. A study of the variability or non-variability of 359 stars (Hall 1991b, 1994) showed that all stars with a Rossby number greater than about 0.65 are insignificantly variable (amplitude less than 0.01 magnitude), and all of the significantly variable stars have a Rossby number less than about 0.65 . Measurable photometric variability, presumed to result from rotational modulation of an unevenly spotted stellar surface, is taken as indication of the presence of large spots. The value 0.65 seems to act as a threshold segregating weak-dynamo from strong-dynamo stars. There may be a second-order effect to be investigated, to explain the range of photometric amplitude among those stars with small Rossby numbers.

At a more basic level, however, we want to know physically what determines the size of the largest spots developed by any star. To my surprise, there seems to have been relatively little work on this question. Let me list three different pictures proposed at one time or another, none of them having ever been demonstrated to be accurate.

Could it be that the full depth of the convective zone determines the diameter of a spot on the surface, a picture first put forth in the early 1970's by Mullan $(1973,1974,1975)$.

Could it be that, under conditions of strong dynamo action, the socalled "giant convective cells" (thought to underlie the photosphere and extend through most of the convection zone's depth) extend to the surface without the usual hierarchical degradation into granular-sized cells.

Could it be that the largest spot size corresponds to the expected supergranulation size, which is considerably smaller than the full depth of the convection zone or the giant convective cells. If this picture is to be right, then the very biggest spots we observe must be groupings of several somewhat smaller, medium-sized spots. See the illuminating discussion by Rucinski (1979).

\section{Starspots as tracers of long-term cycles ?}

Starspots themselves are fated to prove less useful than some other approaches as tracers of long-term cycles akin to the Sun's 11-year sunspot cycle or 22-year magnetic cycle. More successful has been the monitoring of Ca II K-line intensity initiated by O. C. Wilson at Mt. Wilson Observatory. Potentially just as useful is the monitoring of orbital period changes in eclipsing binaries containing a convective star as one component (Hall 1990, 1991a, Applegate 1992). Photometric monitoring of spotted stars has proven less fruitful. Three approaches have been tried. 
The first is to monitor a star's changing mean brightness, in the $\mathrm{V}$ bandpass, for example. First, it is easy to be misled by variability in mean $\mathrm{V}$ magnitude probably associated with the lifetime of a single large spot or a few large spots. Those lifetimes will not be identical to the soughtfor long-term cycles. Second, we know from previous work that, in the Sun and less active stars, more spots coincide (curiously) with a brighter mean $\mathrm{V}$ magnitude. In more active stars, on the other hand, more spots coincide (as expected intuitively) with a fainter mean $\mathrm{V}$ magnitude. This behavior is sure to complicate interpretation of long-term monitoring of mean $\mathrm{V}$ brightness. Third, the expected amplitude of variability in mean $\mathrm{V}$ brightness is not very large, only a few millimagnitudes in less active stars like the Sun and around 0.1 magnitude for more active stars (Applegate 1992). That means we will need a long-term (several decades) baseline of accurately standardized magnitudes, preferably photoelectric.

The second is to monitor a star's changing color, in the B-V color index, for example. The rationale behind this approach is that the variability in luminosity probably arises mostly from a change in surface temperature, not a change in radius. The difficulties here are similar to those expected in monitoring the mean $\mathrm{V}$ brightness, only worse, mainly because the expected color changes are smaller than the expected luminosity changes.

The third is to look for latitude drift, which shows up in the Sun's butterfly diagram as one signature of its 11-year cycle. Doppler imaging has the potential to be the best tool for use in this approach, because it has the power to determine spot latitudes directly. On the other hand, the available baseline of Doppler imaging maps for any one star is quite short, compared to baselines of stars monitored in Ca II K-line emission or broadband photometry. Photometry, despite its superior baseline, must infer latitude drift from a systematic trend in the rotation periods of individual spots, without benefit of direct knowledge of the true rotation-versus-latitude law describing the star's surface differential rotation.

\section{References}

Applegate, J.H. 1992, ApJ 385, 621.

Doyle, J.G., Butler, C.J., Morrison, L.V., and Gibbs, P. 1988, A\&A 192, 275.

Drish, W.F., Harmon, R., Marcialis, R.L., and Wild, W.J. 1995, Icarus 113, 360.

Glebocki, R., Bielicz, E., Pastuszka, Z., and Sikorski, J. 1986, Acta Astr. 36, 369.

Hall, D.S. 1972, PASP 84, 323.

Hall, D.S. 1980, Highlights of Astronomy 5, 841.

Hall, D.S. 1990, in Active Close Binaries, C. Ibanoglu (ed), Dordrecht: Kluwer, p. 95.

Hall, D.S. 1991a, ApJ 380, L85.

Hall, D.S. 1991b, in The Sun and Cool Stars: Activity, Magnetism, Dynamos, I.A.U. Colloq. No. 130, I. Tuominen, D. Moss, and G. Rüdiger (eds), Berlin: Springer-Verlag, p. 353.

Hall, D.S. 1994, Memorie della Societa Astronomica Italiana 65, 73. 
Hall, D.S. and Henry G.W. 1994, I.A.P.P.P. Comm. No. 55, 51.

Hampton, M., Henry, G.W., Eaton, J.A., Nolthenius, R.A., and Hall, D.S. 1995, PASP, submitted.

Henry, G.W., Eaton, J.A., Hamer, J., and Hall, D.S. 1995, ApJS 97, 513.

Mullan, D.J. 1973, ApJ 186, 1059.

Mullan, D.J. 1974, ApJ 192, 149.

Mullan, D.J. 1975, ApJ 198, 563.

Nolthenius, R. 1991, I.B.V.S. No. 3589.

Rodonó, M. 1986, in Cool Stars, Stellar Systems, and the Sun, Fourth Cambridge Workshop, edited by M. Zeilik and D.M. Gibson (Berlin: Springer-Verlag), p.475.

Rucinski, S.M. 1979, Acta Astr. 29, 203.

Wild, W.J. 1994, in Cool Stars, Stellar Systems, and the Sun, Eighth Cambridge Workshop, A.S.P. Conference Series, Vol. 64, edited by J.P. Caillault, p.670.

Zeilik, M. 1992, in Cool Stars, Stellar Systems, and the Sun, Seventh Cambridge Workshop, A.S.P. Conference Series, Vol. 26, edited by M.S. Giampapa and J.A. Bookbinder, p. 346. 